\title{
Size of common bile duct stones on MRCP predicts likelihood of positive findings at ERCP
}

\author{
J. Reid, R. Dolan, M. Patel, R. Fleming, D. Young, A. Hair \\ Department of Surgery, Victoria Infirmary, Langside Road, Glasgow, G42 9TY, UK
}

\begin{abstract}
Objective:

To ascertain if direct measurement of the size of common bile duct stones (CBD) on magnetic resonance cholangio-pancreatography (MRCP) can be used to predict the likelihood of a positive endoscopic retrograde cholangio-pancreatography (ERCP) result. Should we be carrying out ERCPs on all patients with CBD stones?

Methods:
\end{abstract}

We retrospectively analysed the records of 1812 consecutive patients undergoing MRCP between November 2009 and November 2014 at the Victoria Infirmary. A measurable CBD stone was present in 383 patients, of whom 293 successfully underwent ERCP.

Results:

221 patients (75\%) had stones demonstrated on ERCP. A receiver operating curve (ROC) was plotted correlating stone size with the likelihood of a positive ERCP result, and demonstrates that using a cut off of $>4 \mathrm{~mm}$ as an indication for ERCP gives the mathe- matical best-fit correlation with a sensitivity of $83 \%(95 \% \mathrm{Cl} 78,88)$ and specificity of $66 \%(95 \% \mathrm{Cl} 53,77)$.

Conclusions:

In our current practice, all patients with CBD stones at MRCP are considered for ERCP regardless of stone size. Our results would support the hypothesis that as size de- creases the likelihood of spontaneous stone passage increases. Although the threshold of mathematical best compromise is $>4 \mathrm{~mm}$ to minimise both type 1 and type 2 errors, we would favour a lower threshold of $2 \mathrm{~mm}$ above which ERCP is performed (sensitivity 98.65, 95\% Cl 96.1,99.7, specificity $25.71 \%, \mathrm{Cl}$ 16.0,37.6). For patients with stones measuring $2 \mathrm{~mm}$ or less, early operative intervention with intraoperative cholangiography to confirm duct clearance could be a suitable alternative. 


\section{Introduction}

Gallstones are present in approximately $25 \%$ of the UK population at post-mortem, ${ }^{1}$ and complications including common bile duct (CBD) stones account for a significant part of emergency and elective general surgery. ${ }^{1,2}$ The current guidelines issued by the British Society of Gastroenterologists suggest that whenever patients have symptomatic CBD the stones should be removed whenever possible. ${ }^{3}$ They also state that a cholecystectomy should be performed for all patients with CBD stones when accompanied by symptomatic gallstones. ${ }^{1,3}$ The standard sequence of investigation for suspected CBD stones involves transabdominal ultrasound to assess CBD size and plasma liver function tests in the first instance. If either of these is abnormal the next step would be to carry out an MRCP or endoscopic ultrasound. ${ }^{4}$ Both of these tests have a higher sensitivity for the identification of gallstones particularly CBD stones. ${ }^{4-6}$ If stones are confirmed, the current guidelines state that endoscopic retrograde cholangiopancreatography (ERCP) and stone extraction should be performed on all patients fit to undergo the procedure. ${ }^{3}$

ERCP carries significant risks to the patient, with a reported rate of post ERCP pancreatitis as high as $6.7 \%$, gastro-intestinal haemorrhage of between $0.7 \%$ and $2 \%$, cholangitis between $0.5 \%$ and $5 \%$ and duodenal perforation up to $1 \% .^{1,6,7}$ Current guidelines state that patient selection should take into ac- count patient co-morbidities and the likelihood of a negative investigation, and caution against unnecessary biliary instrumentation, stating that ERCP should be reserved for those patients in whom the clinician is confident an intervention will be required. The Joint Advisory Group on Gastrointestinal endoscopy (JAG) recommendations state that $>90 \%$ of $\mathrm{ERCPs}^{8}$ should be performed with therapeutic intent, which is considerably higher than the rate of positive ERCP in our hospital.

In the management of ureteric stones, radiological evaluation of stone size has been shown to clearly correlate with the chance of spontaneous stone passage. ${ }^{1,9}$ While the ureter is of a larger diameter than the CBD and the volume of urine which is a much less adhesive substance than bile is much larger over the course of $24 \mathrm{~h}$ no similar studies looking to correlate stone size to the chance of passing have been carried out. 4,9

As a result, we set out to investigate whether or not CBD stone size measured on MRCP could be used as a predictor of the likelihood of finding CBD stones at ERCP, and thus whether a size 
threshold could be set to avoid ERCP in patients with a high risk of an unnecessary procedure.

\section{Methods}

Clinical records were reviewed over the 60 month period from November 2009 to November 2014 to identify those patients who had a clinical diagnosis of possible CBD stones at a single unit in Glasgow. The results of this review were then correlated with those patients who underwent MRCP scans to ensure that these were for gallstone disease as opposed to another cause. Stone sizes and CBD dilation were reported by a consultant radiologist or a senior registrar via the PACS imaging system.

Those patients who had confirmed MRCP stones were then reviewed to ascertain if they proceeded to ERCP. The reports for each ERCP were reviewed in order to ascertain if these were successful or not and if not the reason why this was the case.

All MRI scans were carried out using a Phillips Achieva 1.5 T MRI scanner. Data was analysed using MedCalc version 13.

All clinical information was stored in an encrypted Microsoft Excel 2007 file. This was in turn stored a secure serve in accordance with local policy in Greater Glasgow and Clyde. Statistical analysis was carried out in SPSS with graph construction also being carried out in SPSS.

Statistical analysis was performed by plotting a receiver operating curve $(R O C)$ of true positive rate (sensitivity) against false negative rate (1-specificity). From this, the sensitivities and specificities of a range of size cut-offs were derived, as well as the value of best mathematical compromise (greatest area under the curve).

\section{Results}

Over the course of the study 1812 consecutive patients with a clinical diagnosis of possible CBD stones underwent MRCP. Stones were demonstrated in 408 patients. Stone size was measured and reported by a consultant radiologist or senior Specialist Trainee Registrar in 383 patients. The remaining 26 were excluded as no measurement was given on the original MRCP report and the images were not available for re-reporting.

73 patients did not undergo ERCP. These patients declined the procedure, were clinically unfit, died prior to ERPC or were otherwise lost to follow-up. ERCP was positive in 221 (75\%) patients. 17 ERCPs failed, and the remaining 72 patients were demonstrated to have a clear duct. Stone size 
ranged from 1 to $32 \mathrm{~mm}$ (median $6 \mathrm{~mm}$ ).

Figure 1 shows the ROC curve quantifying the ability of MRCP measurement of stones to predict ERCP result, with the area under the curve calculated at $0.79(95 \% \mathrm{Cl} 0.74,0.84, \mathrm{p}<0.0001)$.

The point of mathematical best compromise (Youden index $\mathrm{J}$ associated criterion) is shown as stone size $>4 \mathrm{~mm}$.

Table 1 shows criterion values and co-ordinates of the ROC curve, which presents sensitivity and specificity for a variety of thresholds used as a cut-off to decide whether or not to perform ERCP.

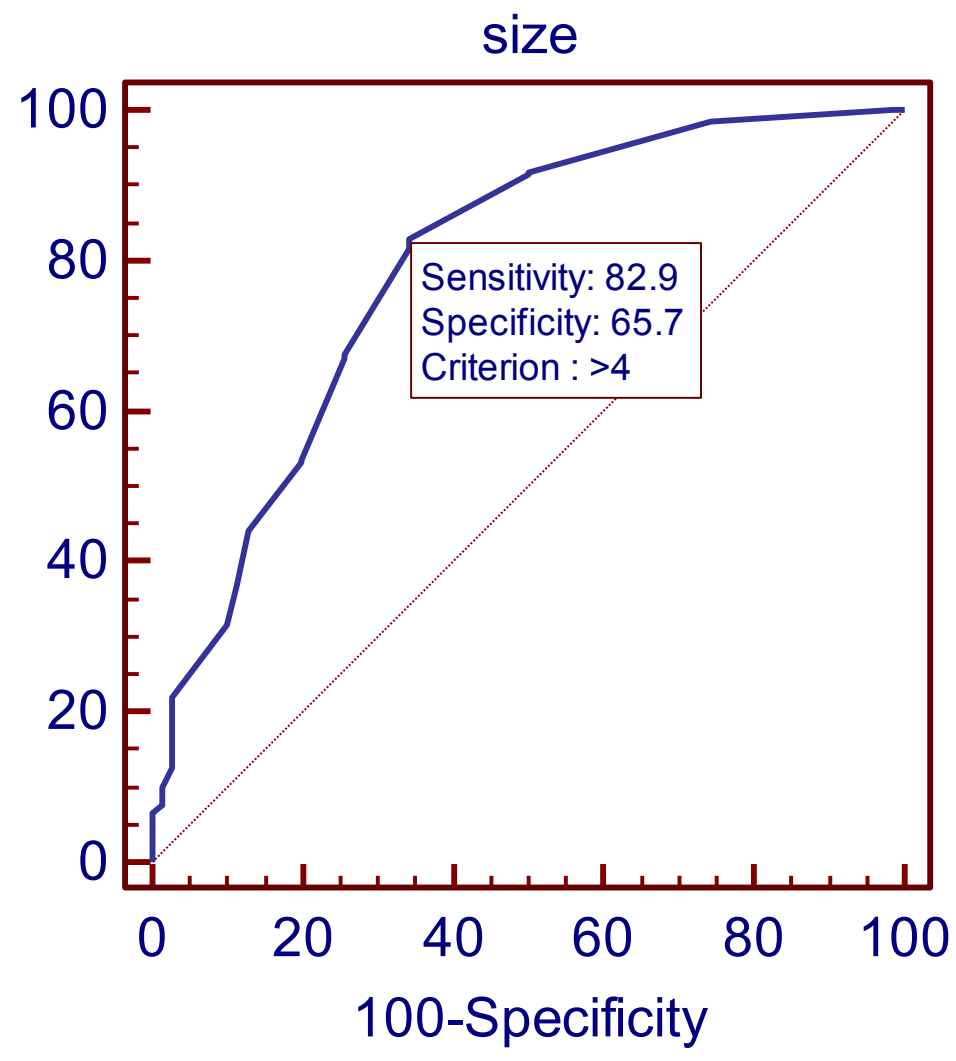

Figure 1: ROC curve showing sensitivity and specificity

\begin{tabular}{|l|r|r|r|r|r|r|}
\hline Criterion & Sensitivity & $95 \% \mathrm{Cl}$ & Specificity & $95 \% \mathrm{Cl}$ & $+\mathrm{LR}$ & -LR \\
\hline$\geq 1$ & 100.00 & $98.4-100.0$ & 0.00 & $0.0-5.1$ & 1.00 & \\
\hline$>1$ & 100.00 & $98.4-100.0$ & 1.43 & $0.04-7.7$ & 1.01 & 0.00 \\
\hline$>2$ & 98.65 & $96.1-99.7$ & 25.71 & $16.0-37.6$ & 1.33 & 0.053 \\
\hline$>3$ & 91.89 & $87.5-95.1$ & 50.00 & $37.8-62.2$ & 1.84 & 0.16 \\
\hline$>3.5$ & 91.44 & $87.0-94.8$ & 50.00 & $37.8-62.2$ & 1.83 & 0.17 \\
\hline$>4$ & 82.88 & $77.3-87.6$ & 65.71 & $53.4-76.7$ & 2.42 & 0.26 \\
\hline
\end{tabular}




\begin{tabular}{|l|l|l|l|l|l|l|}
\hline$>4.5$ & 81.53 & $75.8-86.4$ & 65.71 & $53.4-76.7$ & 2.38 & 0.28 \\
\hline$>5$ & 67.57 & $61.0-73.7$ & 74.29 & $62.4-84.0$ & 2.63 & 0.44 \\
\hline$>5.5$ & 67.12 & $60.5-73.3$ & 74.29 & $62.4-84.0$ & 2.61 & 0.44 \\
\hline$>6$ & 53.60 & $46.8-60.3$ & 80.00 & $68.7-88.6$ & 2.68 & 0.58 \\
\hline$>6.2$ & 53.15 & $46.4-59.9$ & 80.00 & $68.7-88.6$ & 2.66 & 0.59 \\
\hline$>7$ & 44.14 & $37.5-50.9$ & 87.14 & $77.0-93.9$ & 3.43 & 0.64 \\
\hline$>8$ & 36.94 & $30.6-43.7$ & 88.57 & $78.7-94.9$ & 3.23 & 0.71 \\
\hline$>9$ & 31.53 & $25.5-38.1$ & 90.00 & $80.5-95.9$ & 3.15 & 0.76 \\
\hline$>10$ & 22.07 & $16.8-28.1$ & 97.14 & $90.1-99.7$ & 7.73 & 0.80 \\
\hline$>12$ & 12.61 & $8.5-17.7$ & 97.14 & $90.1-99.7$ & 4.41 & 0.90 \\
\hline$>13$ & 9.91 & $6.3-14.6$ & 98.57 & $92.3-100.0$ & 6.94 & 0.91 \\
\hline$>14.5$ & 7.66 & $4.5-12.0$ & 98.57 & $92.3-100.0$ & 5.36 & 0.94 \\
\hline$>15$ & 6.76 & $3.8-10.9$ & 100.00 & $94.9-100.0$ & & 0.93 \\
\hline$>32$ & 0.00 & $0.0-1.6$ & 100.00 & $94.9-100.0$ & & 1.00 \\
\hline
\end{tabular}

Table 1: Criterion values and co-ordinates of the ROC curve

\section{Discussion}

After a detailed literature review we believe that no previous studies have been published examining the relationship between size of CBD stones and their chance of spontaneous passage. Our data suggest that there is a highly significant correlation between increasing stone size and the likelihood of positive ERCP ( $p$ value $<0.0001$ ). This strongly suggests that there is a higher likelihood of smaller stones passing spontaneously prior to attempts at removal via endoscopic means. This is not the standard practice in the majority of centres within the UK and as such we hope will generate considerable debate.

If we were to reduce the number of unnecessary ERCPs, the data would support setting a threshold of stone size, which any given stone must exceed on MRCP measurement before ERCP would be undertaken. ${ }^{4}$ As can be seen in Table 1, as that threshold rises, the specificity of the test rises with it, at the cost of a falling sensitivity. If ERCP is performed for any stone $>1 \mathrm{~mm}$ in size, sensitivity is $100 \%(95 \% \mathrm{Cl} 98.4,100)$ but with a low specificity of $1.43 \%(95 \% \mathrm{Cl}$ $0.04,7.1$ ) and correspondingly high false negative rate - in essence, this is close to our current practice of performing ERCP on all patients with MRCP demonstrated stone disease of any size. 1,4

By contrast, if a threshold of $>15 \mathrm{~mm}$ is set below which ERCP is not undertaken, the sensitivity of the test falls to $6.76 \%(95 \% \mathrm{Cl} 3.8,10.9)$ and the specificity rises to $100 \%(95 \% \mathrm{Cl} 94.9,100)$. Clearly the most useful threshold, at which a good sensitivity and specificity is maintained lies 
somewhere between these two levels.

Mathematically, the point of best compromise (maximal value of Youden's index) sets a threshold of $>4 \mathrm{~mm}$ for undertaking ERCP, with a sensitivity of $82.88 \%(77.3,87.6 \%)$ and specificity of $65.71 \%(53.4,76.7 \%)$ which overall minimises the number of misclassified results. However, the test gives equal weight to the consequences of patients with stones being left untreated and those with a clear duct undergoing unnecessary ERCP, which is not clinically the case. ${ }^{6}$ In practice it would seem more important to accept a lower specificity in order to gain a greater sensitivity, to avoid the consequences of under-treatment. ${ }^{1}$ Even a relatively modest decrease overall in the number of unnecessary ERCPs performed would be an improvement on the current situation, not only reducing the clinical risk to any given patient, but also with implications for waiting lists and resource management if fewer numbers of procedures are performed overall. ${ }^{10,11}$ From these data, we would suggest that a cut off of $>2 \mathrm{~mm}$ in stone size would result in very few stones being missed (sensitivity of 98.65, 95\% Cl 96.1, 99.7), while still correctly identifying a significant number of patients not requiring ERCP (sensitivity $25.71 \%, \mathrm{Cl}$ $16.0,37.6)$.

\section{Conclusions}

In our current practice, all patients with CBD stones at MRCP are considered for ERCP regardless of stone size, which leads to a rate of positive ERCP falling significantly short of the JAG recommendation of $90 \% .8$ Our results support the hypothesis that as size decreases the likelihood of spontaneous stone passage increases, and that measurement of stone size can be used to predict the likelihood of positive ERCP. Although the threshold of mathematical best compromise is $>4 \mathrm{~mm}$ to minimise both Type 1 and Type 2 errors, we would favour a lower threshold of $2 \mathrm{~mm}$ above which ERCP should be performed (sensitivity 98.65, 95\% Cl 96.1, 99.7, sensitivity $25.71 \%, \mathrm{Cl} 16.0,37.6$ ) in order to exclude a significant proportion of patients for where an ERCP is unnecessary. For patients with stones measuring $2 \mathrm{~mm}$ or less, early laparoscopic cholecystectomy with intraoperative cholangiography to confirm duct clearance could be a suitable alternative to ERCP. ${ }^{11}$ A randomised controlled trial is needed to establish efficacy and safety in clinical practice. 
References

1. Khan HN, Harrison M, Bassett EE, Bates TA. 10-year follow-up of a longitudinal study of gallstone prevalence at necropsy in South East England. Dig Dis Sci 2009;54(12):2736e41.

2. Tang E, Stain SC, Tang G, Froes E, Berne TV. Timing of laparoscopic surgery in gallstone pancreatitis. Arch Surg 1995;130(5):496-9.

3. Williams EJ, Green J, Beckingham I, Parks R, Martin D, Lombard M. Guidelines on the management of common bile duct stones (CBDS). Gut 2008;57(7):1004-21.

4. Topal B, Van de Moortel M, Fieuws S, Vanbeckevoort D, Van SW, Aerts R, et al. The value of magnetic resonance cholangiopancreatography in predicting common bile duct stones in patients with gallstone disease. Br J Surg 2003;90(1):42-7.

5. Meduri B, Aubert A, Chiche R, Fritsch J. [Laparoscopic cholecystectomy and lithiasis of the common bile duct: prospective study on the importance of preoperative endoscopic ultrasonography and endoscopic retrograde cholangiography]. Gastroenterol Clin Biol 1998;22(10):759-65.

6. Freeman ML, Nelson DB, Sherman S, Haber GB, Herman ME, Dorsher PJ, et al. Complications of endoscopic biliary sphincterotomy. N Engl J Med 1996;335(13):909-18.

7. Christensen M, Matzen P, Schulze S, Rosenberg J. Complications of ERCP: a prospective study. Gastrointest Endosc 2004;60(5):721-31.

8. Valori R. BSG quality and safety indicators for endoscopy. In: Barton R, editor. Joint advisory group on endoscopy (JAG) 1[2], 1-12. London: JAG Committee; 1-3-2007.

9. Coll DM, Varanelli MJ, Smith RC. Relationship of spontaneous passage of ureteral calculi to stone size and location as revealed by unenhanced helical CT. AJR Am J Roentgenol 2002;178(1):101-3.

10. Lawrentschuk N, Hewitt PM, Pritchard MG. Elective laparoscopic cholecystectomy: implications of prolonged waiting times for surgery. ANZ J Surg 2003;73(11):890-3.

11. Somasekar K, Shankar PJ, Foster ME, Lewis MH. Costs of waiting for gall bladder surgery. Postgrad Med J 2002;78(925):668-9. 